Molecules 2007, 12, 1183-1190

molecules

ISSN 1420-3049

(C) 2007 by MDPI

www.mdpi.org/molecules

Full Paper

\title{
Facile Synthesis of Optically Active Imidazole Derivatives
}

\author{
Ales Marek, Jiri Kulhanek, Miroslav Ludwig and Filip Bures* \\ Department of Organic Chemistry, Faculty of Chemical Technology, University of Pardubice, \\ nam. Cs. legii 565, Pardubice, 53210, Czech Republic \\ * Author to whom correspondence should be addressed; E-mail: filip.bures@upce.cz
}

Received: 11 May 2007; in revised form: 22 May 2007 / Accepted: 23 May 2007 / Published: 30 May 2007

\begin{abstract}
Five optically active imidazole derivatives have been synthesized via a facile 4-step reaction sequence starting from commercially available and inexpensive $\mathrm{N}$-Cbz amino acids. While microwave assisted condensation was unsuccessful, the condensation of the corresponding $\alpha$-bromoketones with formamidine acetate in liquid ammonia was revealed to be a useful method for the synthesis of such imidazole derivatives. The derivatives thus prepared are structurally-related to histamine.
\end{abstract}

Keywords: Imidazole, condensation, optically active ligand

\section{Introduction}

From time immemorial, organic chemists have been attempting to synthesize, isolate and characterize heterocyclic molecules for their unique chemical and physical properties. Despite the fact that many convenient synthetic methods have been utilized for preparing the basic heterocycles [1], a facile synthesis of optically active analogues still remains a challenge. Among the many possible targets enantiopure five-membered heterocyclic derivatives such as imidazole are of particularly interest. For instance, naturally occurring 4-substituted imidazoles such as histidine or histamine and their significance as an essential amino acid or its decarboxylation product, are well known. Imidazole chemistry currently attracts considerable attention, where the imidazole derivatives are widely applied as $N$-ligands coordinating transition metals [2-3]. The application of imidazoles in medicinal chemistry 
[4] or chemistry of natural products/alkaloids [5-6] or of 1,3-disubstituted imidazole salts as ionic liquids [7-8] are also well known. Although a few examples of the synthesis and applications of optically active imidazole derivatives have been published [9-13], development of an efficient synthesis of such derivatives still requires more attention. Recently, we have published the synthesis and application of enantiopure 2-phenylimidazoles starting from the commercially available $\alpha$-amino acids as a source of chirality [14-15]. It would be challenging to synthesize 2,5-unsubstituted imidazoles bearing similar chains with a chiral amine (depending on the starting amino acids used). In addition, optically active imidazole derivatives formed upon cross-coupling reactions at the 2 position with another heterocycles (pyridine, phenanthroline, etc.), in order to further support the ability to bind transition metal ions, might provide interesting enantiopure nitrogen ligands. A possible application of such materials in asymmetric catalysis or as histamine-related products is apparent. Hence, we describe herein the synthesis of the proposed optically active 4-substituted imidazole derivatives as well as their detailed structural analysis.

\section{Results and Discussion}

One of the synthetic pathways used for the construction of the $\mathrm{N}(1)-\mathrm{C}(5)$ and $\mathrm{N}(3)-\mathrm{C}(4)$ bonds of the imidazole ring involves reaction of 1,3-bifunctional electrophiles with various amidines. In order to prepare a 2-unsubstituted imidazole, formamide must be employed, while the 1,3-bifunctional electrophiles could be represented by various haloketones [16-18] or hydroxyketones [20]. Sugar based hydroxyketones or hydroxyaldehydes and their application as suitable 1,3-bifunctional electrophiles are also well known [20-21]. The condensation may be enhanced by microwave irradiation [22] as well. Nevertheless, the most used method for imidazole ring construction involves a condensation of the $\alpha$-bromoketones with formamidine in liquid ammonia [16-21].

During our investigation in the field of enantiopure imidazole synthesis, we have developed an efficient method utilizing $N$-Cbz protected $\alpha$-amino acids $\mathbf{1}$ as a suitable chiral starting material. Activation of the carboxylic function of the $\alpha$-amino acid via mixed anhydrides 2 , followed by its reaction with diazomethane afforded $\alpha$-diazoketones 3 . Treatment of the $\alpha$-diazoketones with hydrobromic acid smoothly provided $\alpha$-bromoketones $\mathbf{4}$, while nitrogen was liberated. In this way prepared $\alpha$-bromoketones may serve as suitable 1,3-bifunctional electrophiles and, using the known condition for condensation in liquid ammonia as described above, we have prepared five optically active imidazole derivatives 5 bearing substituents at position 4 (Scheme 1).

(S)-Cbz-Alanine, (S)-Cbz-valine, (S)-Cbz-leucine, (S)-Cbz-isoleucine and (S)-Cbz-phenylalanine were used as the starting $N$-Cbz protected $\alpha$-amino acids. The condensation reactions of the $\alpha$-bromoketones with formamidine acetate were carried out in a pressure vessel containing liquefied ammonia at $70{ }^{\circ} \mathrm{C}$. The reaction mixture was stirred overnight, extracted and purified by column chromatography to afford pure imidazoles 5a-e. Yields and selected physical properties of the compounds prepared are summarized in the Table 1. 


\section{Scheme 1.}
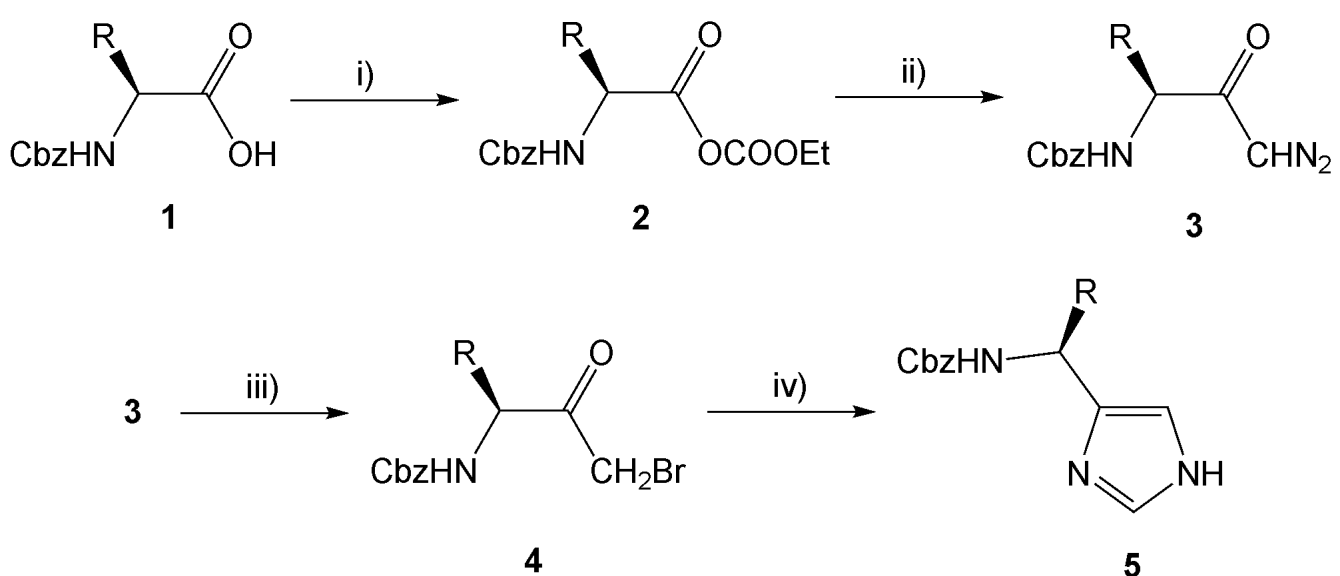

i) $\mathrm{ClCO}_{2} \mathrm{Et} / \mathrm{Et}_{3} \mathrm{~N} / \mathrm{Et}{ }_{2} \mathrm{O} / \mathrm{THF} / 25^{\circ} \mathrm{C} / 30 \mathrm{~min}$; ii) $\mathrm{CH}_{2} \mathrm{~N}_{2} / \mathrm{Et}_{2} \mathrm{O} /-10^{\circ} \mathrm{C} / 3 \mathrm{~h}$; iii) $\mathrm{HBr} / \mathrm{AcOH} / 20^{\circ} \mathrm{C} / 1 \mathrm{~h}$; iv) formamidine acetate $/ \mathrm{NH}_{3}(\mathrm{I}) / 23 \mathrm{bar} / 70^{\circ} \mathrm{C} / 20 \mathrm{~h}$.

Table 1.

\begin{tabular}{ccccc}
\hline Comp. & $\mathbf{R} /$ Starting amino acid & Yield $^{[\mathbf{a}]}[\mathbf{\%}]$ & e.e. [\%] & {$[\boldsymbol{\alpha}]_{\mathbf{D}}{ }^{\mathbf{2 0}}$} \\
\hline $\mathbf{5 a}$ & $\mathrm{CH}_{3} /(S)$-Alanine & $81 / 69$ & 99 & $-15.8(c 1, \mathrm{MeOH})$ \\
$\mathbf{5 b}$ & $\left(\mathrm{CH}_{3}\right)_{2} \mathrm{CH} /(S)$-Valine & $49 / 42$ & 99 & $-31.7(c 0.52, \mathrm{MeOH})$ \\
$\mathbf{5 c}$ & $\left(\mathrm{CH}_{3}\right)_{2} \mathrm{CHCH}_{2} /(S)$-Leucine & $69 / 57$ & 99 & $-28.5(c 0.46, \mathrm{MeOH})$ \\
$\mathbf{5 d}$ & $\mathrm{CH}_{3} \mathrm{CH}_{2}\left(\mathrm{CH}_{3}\right) \mathrm{CH} /(S)-$ Isoleucine & $72 / 56$ & 99 & $-35.6(c 1, \mathrm{MeOH})$ \\
$\mathbf{5 e}$ & $\mathrm{PhCH}_{2} /(S)-\mathrm{Phenylalanine}$ & $40 / 37$ & 99 & $-29.0(c 1, \mathrm{MeOH})$ \\
\hline
\end{tabular}

[a] GC/isolated yields of the final condensation reaction

The enantiomeric excesses (e.e.) were determined by ${ }^{1} \mathrm{H}$-NMR spectroscopy using $(R)$-Mosher's acid. The resulting spectra were compared with the spectra obtained from the corresponding racemate. No racemization was observed throughout the entire reaction sequence. All of the synthesized imidazoles were further characterized by ${ }^{1} \mathrm{H}$ - and ${ }^{13} \mathrm{C}-\mathrm{NMR}$ spectroscopy, EI-MS and elemental analysis (see Experimental section). The NMR structural analyses of the ligands showed the presence of strong hydrogen bonds in a common $\mathrm{CDCl}_{3}$ solution, which resulted in hindered imidazole tautomerism and hindered rotation in the protecting carbamate function used. ${ }^{1} \mathrm{H}$ - and ${ }^{13} \mathrm{C}-\mathrm{NMR}$ spectra then showed broad signals or two set of signals, without expected spin-spin interactions. The hindered imidazole tautomerism and rotation in the carbamate function was suppressed if measured in deuteromethanol. Hence, the ${ }^{1} \mathrm{H}$ - and ${ }^{13} \mathrm{C}$-NMR spectra listed in the Experimental were measured in $\mathrm{CD}_{3} \mathrm{OD}$.

In addition to the condensation reaction carried out in liquid ammonia, microwave assisted reaction [22] was attempted as well. The $\alpha$-hydroxyketones $\mathbf{6}$, obtained from the conversion of the $\alpha$-diazoketone 3 using hydrochloric acid [23], were treated with formamidine acetate and irradiated with microwaves (Scheme 2). Only traces of one corresponding imidazole 5a could be isolated, even 
after we tried to optimize the reaction conditions. It is also noteworthy that no formation of the corresponding imidazole was observed if another $\alpha$-hydroxy- or $\alpha$-bromoketone used. Hence, the microwave assisted reaction was shown to be unsuitable for our derivatives.

Scheme 2.

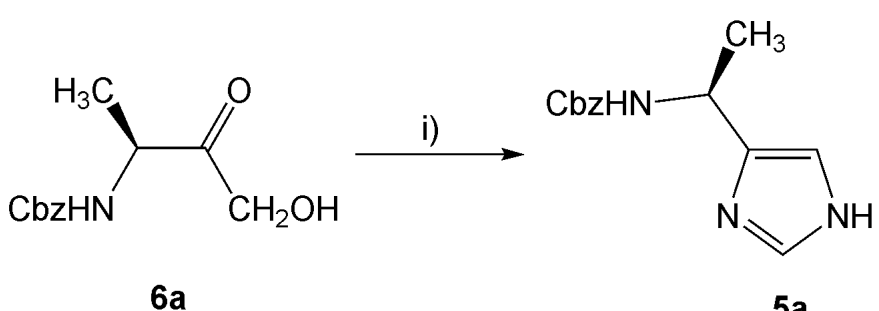

$6 a$

$5 \mathbf{a}$

i) formamidine acetate $/ \mathrm{H}_{2} \mathrm{O} / \mathrm{MW}$ irradiation $2 \times 2 \min (360 \mathrm{~W}) /$ yield $7.5 \%$

\section{Conclusions}

Five novel imidazole derivatives have been synthesized and isolated in satisfactory yields (69$37 \%)$. The crucial step of the reaction sequence was shown to be the condensation of the corresponding $\alpha$-bromoketones with formamidine acetate in liquid ammonia. In $\mathrm{CDCl}_{3}$ solutions the products showed hindered imidazole tautomerism and also hindered carbamate function rotation, which makes the NMR analysis difficult and, therefore, the spectra were measured in deuteromethanol. Microwave assisted reaction of the $\alpha$-hydroxyketones was revealed to be incompatible with the above discussed intermediates. Overall, we have developed a facile 4-step synthesis of histamine-related imidazole derivatives starting from the commercially available and inexpensive $\mathrm{N}$-Cbz amino acids. Cross-couplings of the synthesized imidazoles with other heterocycles, in order to produce even larger nitrogen ligands with stronger coordinating ability, are now under investigation.

\section{Experimental}

\section{General}

Reagents and solvents (reagent grade) were purchased from Aldrich or Fluka and used as received. The $\alpha$-bromoketones $\mathbf{4}$ and $\alpha$-hydroxyketones $\mathbf{6}$ were synthesized according to the literature procedures [14, 23]. Evaporation and concentration in vacuo were performed at water aspirator pressure. The condensation reactions in liquid ammonia were carried out in a ROTH pressure vessel. The microwave assisted reactions were conducted in a MILESTONE MLS ETHOS 1600 URM oven (300 W; $2.45 \mathrm{GHz}$ ). Column chromatography (CC) was carried out with $\mathrm{SiO}_{2} 60$ (particle size 0.040$0.063 \mathrm{~mm}, 230-400$ mesh; Merck) and commercially available solvents. Thin-layer chromatography (TLC) was conducted on aluminium sheets coated with $\mathrm{SiO}_{2} 60 \mathrm{~F}_{254}$ obtained from Merck, with visualization by UV lamp (254 or $360 \mathrm{~nm}$ ). Melting points (M.p.) were measured on a Buchi B-540 melting-point apparatus in open capillaries and are uncorrected. ${ }^{1} \mathrm{H}$ - and ${ }^{13} \mathrm{C}$-NMR spectra were recorded in $\mathrm{CD}_{3} \mathrm{OD}$ at $360 \mathrm{MHz}$ or $90 \mathrm{MHz}$, respectively, with Bruker $\mathrm{AMX} 360$ instrument at $20{ }^{\circ} \mathrm{C}$. Chemical shifts are reported in ppm relative to the signal of $\mathrm{Me}_{4} \mathrm{Si}$. Residual solvent signals in the ${ }^{1} \mathrm{H}$ - 
and ${ }^{13} \mathrm{C}$-NMR spectra were used as an internal reference $\left(\mathrm{CD}_{3} \mathrm{OD}-3.31\right.$ and $49.15 \mathrm{ppm}$ for ${ }^{1} \mathrm{H}$ - and ${ }^{13} \mathrm{C}-\mathrm{NMR}$, respectively). Coupling constants $(J)$ are given in $\mathrm{Hz}$. The apparent resonance multiplicity is described as s (singlet), br s (broad singlet), d (doublet), dd (doublet of doublets), t (triplet), q (quartet) and m (multiplet). Optical rotation values were measured on a Perkin Elmer 341 instrument, concentration $c$ is given in $\mathrm{g} / 100 \mathrm{~mL} \mathrm{CH}_{3} \mathrm{OH}$. The mass spectra were measured on GC/MS configuration comprised of an Agilent Technologies - 6890N gas chromatograph (HP-5MS column, length 30 m, I.D. 0.25 mm, film $0.25 \mu \mathrm{m}$ ) equipped with a 5973 Network MS detector (EI $70 \mathrm{eV}$, mass range 33-550 Da).

\section{General method for the condensations in liquid ammonia}

Into a cooled $\left(-78^{\circ} \mathrm{C}\right)$ pressure vessel equipped with a magnetic stirrer, ammonia $(100 \mathrm{~mL})$ was liquefied, followed by addition of the appropriate $\alpha$-bromoketone $(6.3 \mathrm{mmol})$ and formamidine acetate ( $0.65 \mathrm{~g} ; 6.3 \mathrm{mmol}$ ). The reaction mixture was stirred $20 \mathrm{~h}$ at $70{ }^{\circ} \mathrm{C}$ at $23-26 \mathrm{bar}$, the residual ammonia evaporated, the residue taken up in $\mathrm{CHCl}_{3}(100 \mathrm{~mL})$ and the organic extract washed successively with sat. aq. potassium carbonate $(3 \times 100 \mathrm{~mL})$ and water $(200 \mathrm{~mL})$. The organic layer was dried $\left(\mathrm{Na}_{2} \mathrm{SO}_{4}\right)$ and evaporated in vacuo. $\mathrm{CC}\left(\mathrm{SiO}_{2} ; \mathrm{EtOAc} /\right.$ hexane $1: 1$ to $\mathrm{CHCl}_{3} / \mathrm{CH}_{3} \mathrm{OH} / \mathrm{NH}_{4} \mathrm{OH}$ 5:1:0.1) afforded the pure products.

(S)-Benzyl 1-(1H-imidazol-4-yl)ethylcarbamate (5a). Synthesized from $\alpha$-bromoketone 4a (69 \% yield) as a white solid; $\mathrm{R}_{\mathrm{f}}=0.55\left(\mathrm{SiO}_{2} ; \mathrm{CHCl}_{3} / \mathrm{CH}_{3} \mathrm{OH} / \mathrm{NH}_{4} \mathrm{OH}\right.$ 5:1:0.1); M.p. $119-121{ }^{\circ} \mathrm{C} ;[\alpha]_{\mathrm{D}}{ }^{20}=-$ 15.8 (с 1, $\left.\mathrm{CH}_{3} \mathrm{OH}\right) ;{ }^{1} \mathrm{H}-\mathrm{NMR}: \delta=1.46$ (3H, d, $J=6.9, \mathrm{CH}_{3}$ ), 4.81 (1H, q, $\left.J=6.9, \mathrm{CH}\right), 5.04-5.11(2 \mathrm{H}$, m, $\left.\mathrm{PhCH}_{2}\right), 6.90\left(1 \mathrm{H}, \mathrm{s}, 4-\mathrm{H}_{\mathrm{im}}\right), 7.27-7.34(5 \mathrm{H}, \mathrm{m}, \mathrm{Ar}), 7.59\left(1 \mathrm{H}, \mathrm{d}, J=1.2,2-\mathrm{H}_{\mathrm{im}}\right) ;{ }^{13} \mathrm{C}-\mathrm{NMR}: \delta=$ $21.32\left(\mathrm{CH}_{3}\right), 45.92(\mathrm{CH}), 67.53\left(\mathrm{PhCH}_{2}\right), 116.27$ (5- $\left.\mathrm{C}_{\mathrm{im}}\right), 128.95$ (Ar), 129.09 (Ar), 129.57 (Ar), 136.48 (2-C $\mathrm{im}$ ), 138.50 (Ar), 141.9 (4-C $\mathrm{im}$ ), 158.53 (CO); EI-MS (70 eV) m/z (\%): 245 (1), 154 (10), 137 (10), 122 (50), 108 (90), 94 (60), 79 (100), 67 (10), 51 (20), 44 (70); Elemental analysis (\%) calcd. for $\mathrm{C}_{13} \mathrm{H}_{15} \mathrm{~N}_{3} \mathrm{O}_{2}$ (245.12): C 63.66, H 6.16, N 17.13; found: C 63.66, H 6.15, N 17.06.

(S)-Benzyl 1-(1H-imidazol-4-yl)-2-methylpropylcarbamate (5b). Synthesized from $\alpha$-bromoketone $\mathbf{4 b}$ (42 \% yield) as an amorphous solid; $\mathrm{R}_{\mathrm{f}}=0.61\left(\mathrm{SiO}_{2} ; \mathrm{CHCl}_{3} / \mathrm{CH}_{3} \mathrm{OH} / \mathrm{NH}_{4} \mathrm{OH}\right.$ 5:1:0.1); M.p. 95-100 ${ }^{\circ} \mathrm{C}$; $[\alpha]_{\mathrm{D}}{ }^{20}=-31.7$ (c 0.52, $\left.\mathrm{CH}_{3} \mathrm{OH}\right) ;{ }^{1} \mathrm{H}-\mathrm{NMR}: \delta=0.86\left(3 \mathrm{H}, \mathrm{d}, J=6.7, \mathrm{CH}_{3}\right), 0.92(3 \mathrm{H}, \mathrm{d}, J=6.7$, $\left.\mathrm{CH}_{3}\right), 2.07-2.16(1 \mathrm{H}, \mathrm{m}, \mathrm{CH}), 4.50(1 \mathrm{H}, \mathrm{d}, J=7.2, \mathrm{NCH}), 5.03-5.11\left(2 \mathrm{H}, \mathrm{m}, \mathrm{PhCH}_{2}\right), 6.93$ (1H, s, 4$\mathrm{H}_{\mathrm{im}}$ ), 7.25-7.35 (5h, m, Ar), 7.64 (1H, d, $\left.J=1.1,2-\mathrm{H}_{\mathrm{im}}\right) ;{ }^{13} \mathrm{C}-\mathrm{NMR}: \delta=18.94\left(\mathrm{CH}_{3}\right), 20.15\left(\mathrm{CH}_{3}\right)$, $33.92(\mathrm{CH}), 56.26(\mathrm{NCH}), 67.61\left(\mathrm{PhCH}_{2}\right), 117.19\left(5-\mathrm{C}_{\mathrm{im}}\right), 128.90(\mathrm{Ar}), 129.09(\mathrm{Ar}), 129.58(\mathrm{Ar})$, 136.25 (2-C $\mathrm{C}_{\mathrm{im}}$ ), 138.53 (Ar), 140.04 (4-C $\mathrm{im}_{\mathrm{m}}$ ), 158.68 (CO); EI-MS (70 eV) m/z (\%): 274 (20), 230 (20), 166 (100), 136 (10), 122 (10), 108 (30), 91 (30), 79 (30), 53 (10), 45 (70); Elemental analysis (\%) calcd. for $\mathrm{C}_{15} \mathrm{H}_{19} \mathrm{~N}_{3} \mathrm{O}_{2}$ (273.15): C 65.91, H 7.01, N 15.37; found: C 65.91, H 7.01, N 15.21.

(S)-Benzyl 1-(1H-imidazol-4-yl)-3-methylbutylcarbamate (5c). Synthesized from $\alpha$-bromoketone 4c (57 \% yield) as an amorphous solid; $\mathrm{R}_{\mathrm{f}}=0.63\left(\mathrm{SiO}_{2} ; \mathrm{CHCl}_{3} / \mathrm{CH}_{3} \mathrm{OH} / \mathrm{NH}_{4} \mathrm{OH}\right.$ 5:1:0.1); M.p. 32-36 ${ }^{\circ} \mathrm{C}$; $[\alpha]_{\mathrm{D}}{ }^{20}=-28.5\left(\mathrm{c} 1, \mathrm{CH}_{3} \mathrm{OH}\right) ;{ }^{1} \mathrm{H}-\mathrm{NMR}: \delta=0.93\left(6 \mathrm{H}, \mathrm{d}, J=6.1,2 \times \mathrm{CH}_{3}\right), 1.61-1.70(3 \mathrm{H}, \mathrm{m}, \mathrm{CH}+$ $\left.\mathrm{CH}_{2}\right), 4.78(1 \mathrm{H}, \mathrm{t}, J=7.9, \mathrm{NCH}), 5.05-5.11\left(2 \mathrm{H}, \mathrm{m}, \mathrm{PhCH}_{2}\right), 6.90\left(1 \mathrm{H}, \mathrm{s}, 4-\mathrm{H}_{\mathrm{im}}\right), 7.24-7.33(5 \mathrm{H}, \mathrm{m}$, 
Ar), $7.58\left(1 \mathrm{H}, \mathrm{d}, J=1.2,2-\mathrm{H}_{\mathrm{im}}\right) ;{ }^{13} \mathrm{C}-\mathrm{NMR}: \delta=22.55\left(\mathrm{CH}_{3}\right), 23.38\left(\mathrm{CH}_{3}\right), 26.17\left(\mathrm{CH}_{2}\right), 45.42(\mathrm{CH})$, 48.44 (CH), $67.53\left(\mathrm{PhCH}_{2}\right), 116.60$ (5- $\left.\mathrm{C}_{\mathrm{im}}\right), 128.89$ (Ar), 129.07 (Ar), 129.57 (Ar), 136.43 (2-Cim), 138.55 (Ar), 141.44 (4-C $\mathrm{C}_{\mathrm{m}}$ ), 158.42 (CO); EI-MS (70 eV) m/z (\%): 287 (1), 230 (20), 196 (10), 186 (20), 136 (10), 122 (100), 108 (50), 91 (60), 79 (60), 67 (10), 51 (10), 39 (10); Elemental analysis (\%) calcd. for $\mathrm{C}_{16} \mathrm{H}_{21} \mathrm{~N}_{3} \mathrm{O}_{2}$ (287.16): C 66.88, H 7.37, N 14.62; found: C 66.64, H 7.34, N 14.55.

(1S,2R)-Benzyl-1-(1H-imidazol-4-yl)-2-methylbutylcarbamate (5d). Synthesized from $\alpha$-bromoketone 4d (56 \% yield) as an amorphous solid; $\mathrm{R}_{\mathrm{f}}=0.60\left(\mathrm{SiO}_{2} ; \mathrm{CHCl}_{3} / \mathrm{CH}_{3} \mathrm{OH} / \mathrm{NH}_{4} \mathrm{OH}\right.$ 5:1:0.1); M.p. 45-49 ${ }^{\circ} \mathrm{C} ;[\alpha]_{\mathrm{D}}{ }^{20}=-35.6$ (c 0.46, $\left.\mathrm{CH}_{3} \mathrm{OH}\right) ;{ }^{1} \mathrm{H}-\mathrm{NMR}: \delta=0.81$ (3H, d, $\left.J=6.7, \mathrm{CH}_{3}\right), 0.90$ (3H, t, $J=7.3$, $\left.\mathrm{CH}_{3}\right), 1.15+1.54\left(2 \mathrm{H}, 2 \times \mathrm{m}, \mathrm{CH}_{2}\right), 1.87-1.94(1 \mathrm{H}, \mathrm{m}, \mathrm{CH}), 4.57(1 \mathrm{H}, \mathrm{d}, J=7.3, \mathrm{NCH}), 5.00-5.10$ $\left(2 \mathrm{H}, \mathrm{m}, \mathrm{PhCH}_{2}\right), 6.91$ (1H, s, 4- $\left.\mathrm{H}_{\mathrm{im}}\right), 7.25-7.32(5 \mathrm{H}, \mathrm{m}, \mathrm{Ar}), 7.60\left(1 \mathrm{H}, \mathrm{d}, J=0.8,2-\mathrm{H}_{\mathrm{im}}\right) ;{ }^{13} \mathrm{C}-\mathrm{NMR}: \delta$ $=11.79\left(\mathrm{CH}_{3}\right), 16.41\left(\mathrm{CH}_{3}\right), 26.28\left(\mathrm{CH}_{2}\right), 40.33(\mathrm{CH}), 55.08(\mathrm{CH}), 67.57\left(\mathrm{PhCH}_{2}\right), 117.31\left(5-\mathrm{C}_{\mathrm{im}}\right)$,

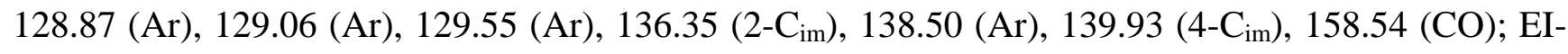
MS (70 eV) m/z (\%): 287 (1), 230 (20), 186 (20), 136 (20), 122 (100), 108 (70), 91 (60), 79 (70), 67 (20), 51 (20), 39 (20); Elemental analysis (\%) calcd. for $\mathrm{C}_{16} \mathrm{H}_{21} \mathrm{~N}_{3} \mathrm{O}_{2}$ (287.16): C 66.88, H 7.37, N 14.62; found: C 66.70, H 7.25, N 14.62.

(S)-Benzyl 1-(1H-imidazol-4-yl)-2-phenylethylcarbamate (5e). Synthesized from $\alpha$-bromoketone 4e (37\% yield) as a brown solid; $\mathrm{R}_{\mathrm{f}}=0.65\left(\mathrm{SiO}_{2} ; \mathrm{CHCl}_{3} / \mathrm{CH}_{3} \mathrm{OH} / \mathrm{NH}_{4} \mathrm{OH}\right.$ 5:1:0.1); M.p. 138-140 ${ }^{\circ} \mathrm{C}$; $[\alpha]_{\mathrm{D}}{ }^{20}=-29.0\left(\right.$ c $\left.1, \mathrm{CH}_{3} \mathrm{OH}\right) ;{ }^{1} \mathrm{H}-\mathrm{NMR}: \delta=2.99\left(1 \mathrm{H}, \mathrm{dd},{ }^{2} \mathrm{~J}(\mathrm{H}, \mathrm{H})=13.6,{ }^{3} \mathrm{~J}(\mathrm{H}, \mathrm{H})=8.8, \mathrm{CH}_{2}\right), 3.20$ $\left(1 \mathrm{H}, \mathrm{dd},{ }^{2} J(\mathrm{H}, \mathrm{H})=13.6,{ }^{3} J(\mathrm{H}, \mathrm{H})=6.1, \mathrm{CH}_{2}\right), 3.92-5.05\left(4 \mathrm{H}, \mathrm{m}, \mathrm{CH}+\mathrm{NH}+\mathrm{CH}_{2}-\right.$ merged with residual $\left.\mathrm{CH}_{3} \mathrm{OH}\right), 6.85\left(1 \mathrm{H}, \mathrm{s}, 4-\mathrm{H}_{\mathrm{im}}\right), 7.11-7.32(10 \mathrm{H}, \mathrm{m}, 2 \times \mathrm{Ar}), 7.62\left(1 \mathrm{H}, \mathrm{d}, J=1.1,2-\mathrm{H}_{\mathrm{im}}\right) ;{ }^{13} \mathrm{C}-$ NMR: $\delta=42.50\left(\mathrm{CH}_{2}\right), 52.21(\mathrm{NCH}), 67.41\left(\mathrm{PhCH}_{2}\right), 116.42\left(5-\mathrm{C}_{\mathrm{im}}\right), 127.48$ (Ar), $128.74(\mathrm{Ar})$, 128.99 (Ar), 129.35 (Ar), 129.55 (Ar), 130.53 (Ar), 136.52 (2-C $\mathrm{C}_{\mathrm{im}}$ ), 138.52 (Ar), 139.77 (4-Cim), 141.59 (Ar), 158.26 (CO); EI-MS (70 eV) m/z (\%): 230 (10), 213 (20), 169 (80), 142 (30), 122 (100), 115 (30), 108 (60), 91 (60), 79 (60), 65 (20), 51 (20). Elemental analysis (\%) calcd. for $\mathrm{C}_{19} \mathrm{H}_{21} \mathrm{~N}_{3} \mathrm{O}_{2}$ (321.15): C 71.01, H 5.96, N 13.08; found: C 70. 97, H 5.96, N 13.08.

\section{Microwave assisted condensation}

A few drops of water were added to a mixture of $6 \mathbf{a}(0.2 \mathrm{~g}$; $0.84 \mathrm{mmol})$ and formamidine acetate ( $0.17 \mathrm{~g} ; 1.68 \mathrm{mmol}$ ), which was then treated for $2 \times 2 \mathrm{~min}$ in a $360 \mathrm{~W}$ microwave oven. The resulting mass was taken up in $\mathrm{CHCl}_{3}(10 \mathrm{~mL})$, washed successively with water $(2 \times 10 \mathrm{~mL})$ and concentrated in vacuo. The crude product was purified in the same way as described above for the condensation in liquid ammonia. Yield $7.5 \%$.

\section{Acknowledgements}

This research was supported by the Ministry of Education, Youth and Sport (MSM 0021627501) and by the Czech Science Foundation (203/07/P013). 


\section{References}

1. Katritzky A. R.; Pozharskii A. F. Handbook of Heterocyclic Chemistry; $2^{\text {nd }}$ Edition; Pergamon: New York, 2000.

2. Kamaraj K.; Kim E.; Galliker B.; Zakharov L. N.; Rheingold A. R.; Zuberbuhler A. D.; Karlin K. D. Copper(I) and copper(II) complexes possessing cross-linked imidazole-phenol ligands: Structures and dioxygen reactivity. J. Am. Chem. Soc. 2003, 15, 6028-6029.

3. Moore L. R.; Cooks S. M.; Anderson M. S.; Schanz H.-J.; Griffin S. T.; Rogers R. D.; Kirk M. C.; Shaughnessy K. H. Synthesis and characterization of water-soluble silver and palladium imidazol-2-ylidene complexes with noncoordinating anionic substituents. Organometallics 2006, 25, 5151-5158.

4. Wiglenda T.; Gust R. Structure-Activity Relationship study to understand the estrogen receptordependent gene activation of aryl- and alkyl-substituted $1 \mathrm{H}$-imidazoles. J. Med. Chem. 2007, 50, 1475-1484.

5. Baran P. S.; O’Malley D. P.; Zografos A. L. Sceptrin as a potential biosynthetic precursor to complex pyrrole-imidazole alkaloids: The total synthesis of ageliferin. Angew. Chem. Int. Ed. 2004, 43, 2674-2677.

6. O’Malley D. P.; Li K.; Maue M.; Zografos A. L.; Baran P. S. Total synthesis of dimeric pyrroleimidazole alkaloids: Sceptrin, ageliferin, nagelamide E, oxysceptrin, nakamuric acid, and the axinellamine carbon skeleton. J. Am. Chem. Soc. 2007, 129, 4762-4775.

7. Wang R.; Xiao J.-C.; Twamley B.; Shreeve J. M. Efficient Heck reactions catalyzed by a highly recyclable palladium(II) complex of a pyridyl-functionalized imidazolium-based ionic liquid. Org. Biomol. Chem. 2007, 5, 671-678.

8. Kan H.-C.; Tseng M.-C.; Chu Y.-H. Bicyclic imidazolium-based ionic liquids: Synthesis and characterization. Tetrahedron 2007, 63, 1644-1653.

9. Yang C.-G.; Wang J.; Jiang B. Enantioselective synthesis of the aminoimidazole segment of Dragmacidin D. Tetrahedron Lett. 2002, 43, 1063-1066.

10. Santagostini L.; Gullotti M.; Pagliarin R.; Bianchi E.; Casella L.; Monzani E. Functional mimics of copper enzymes. Synthesis and stereochemical properties of the copper(II) complexes of a trinucleating ligand derived from L-Histidine. Tetrahedron: Asymmetry 1999, 10, 281-295.

11. You J.-S.; Yu X.-Q.; Zhang G.-L.; Xiang Q.-X.; Lan J.-B.; Xie R.-G. Novel chiral imidazole cyclophane receptors: synthesis and enantioselective recognition for amino acid derivatives. Chem. Commun. 2001, 1816-1817.

12. Suwinski J.; Szczepankiewicz W.; Swierczek K.; Walczak K. Synthesis of chiral imidazole derivatives as purine precursors. Eur. J. Org. Chem. 2003, 1080-1084.

13. Jiang H.-Y.; Zhou C.-H.; Luo K.; Chen H.; Lan J.-B.; Xie R.-G. Chiral imidazole metalloenzyme models: Synthesis and enantioselective hydrolysis for $\alpha$-amino acid esters. J. Mol. Catal. A Chem. 2006, 260, 288-294.

14. Bures F.; Kulhanek J. Chiral imidazole derivatives synthesis from enantiopure $N$-protected $\alpha$-amino acids. Tetrahedron: Asymmetry 2005, 16, 1347-1354. 
15 Bures F.; Szotkowski T.; Kulhanek J.; Pytela O.; Ludwig M.; Holcapek M. Novel nitrogen ligands based on imidazole derivatives and their application in asymmetric catalysis. Tetrahedron: Asymmetry 2006, 17, 900-907.

16. Sellier C.; Buschauer A.; Elz S.; Schunack W. Synthesis of (Z)- and (E)-3-(1H)-imidazol-4-yl)-2propenamine and some 3-(1H-imidazol-4-yl)propanamines. Liebigs Ann. Chem. 1992, 317-323.

17. Leschke C.; Altman J.; Schunack W. Bis[1H-imidazol-4(5)-yl]ethane and bis(1-tritylimidazol-4yl)alkanes. Synthesis 1993, 197-198.

18. Elz S.; Schunack W. An alternative synthesis of Homohistamine and structurally related (imidazol-4-yl)alkylamines. Z. Naturforsch. 1987, 42b, 238-242.

19. Griffith R. K.; DiPietro R. A. An improved preparation of imidazole-4(5)-methanol hydrochloride. Synthesis 1983, 576.

20. Siendt H.; Tschamber T.; Streith J. Improved double epimerisation of (D)-glucose into (D)-gulose and the synthesis of (D)-xylo-imidazolopiperidinose. Tetrahedron Lett. 1999, 40, 5191-5192.

21. Streith J.; Boiron A.; Frankowski A.; Le Nouen D.; Rudyk H.; Tschamber T. On the way to glycoprocessing inhibotors: A general one-pot synthesis of imidazolosugars. Synthesis 1995, 944946.

22. Tschamber T.; Rudyk H.; Le Nouen D. Expeditious syntheses of imidazole $C$-nucleosides $(=C$ glycosylimidazoles) from carbohydrates and formamidine acetate. Helv. Chim. Acta 1999, 82, 2015-2019.

23. Ramtohul Y. K.; James M. N. G.; Vederas J. C. Synthesis and evaluation of keto-glutamine analogues as inhibitors of Hepatitis A virus 3C proteinase. J. Org. Chem. 2002, 67, 3169-3178.

Sample Availability: Samples of the compounds 5a-e are available from the authors.

(C) 2007 by MDPI (http://www.mdpi.org). Reproduction is permitted for noncommercial purposes. 